\title{
THE EFFECTS OF CHAIR- BASED EXERCISES ON BALANCE, MUSCLE STRENGTH AND LIFE QUALITY IN FRAIL COMMUNITY- DWELLING OLDER PEOPLE
}

Author: Vincenta Rimonytè

Research supervisor: lecturer doctor Asta Mastavičiūtė

The Department of Rehabilitation, Physical and Sports Medicine, Faculty of Medicine, Vilnius

University, Lithuania

Vilnius University Hospital Santaros klinikos, Rehabilitation, Physical and Sports Medicine Centre

Aim: To determine the efficiency of chair-based exercises on balance, muscle strength and life quality in frail communitydwelling older people.

Introduction: Many older people have difficulties to participate in high intensity physical training, therefore chair-based exercise program may could be used as safe alternative, which could help to stay functional independent longer and avoid worsening of frailty. However, data about chair-based exercises in frail elderly are not sufficient *

Materials and methods: The study was conducted in Vilnius social care home, from October, 2016 till April, 2017. Forty two frail elderly people had participated in the study. 5 Frailty criteria (by L.Fried) were inclusion criteria for this research (exhaustion, unintentional weight loss, muscle weakness, slowness while walking, and low levels of activity) Fig. 1)). The average age of participants was 81.93 years. Participants were divided into two groups: experimental - chair-based exercises $(n=21)$ and control $(n=21)$ - which was occupied with their ordinary activities (stationary bike, walking, etc.). Balance of participants was assessed by the Berg Balance Scale (BBS), Functional Reach Test (FRT), Timed Up and Go Test (TUG). The legs muscle strength was assessed by Sit-to-Stand Test (SST), hand muscle strength was assessed using mechanical dynamometer. Quality of life was evaluated using SF-36 Questionnaire. Statistical data analysis was carried out using SPSS 23.0 for Windows and Microsoft Excel 2013 programs.

Results: The results of SST, TUG, hand and legs muscle strength parameters showed statistically significant improvement in the experimental group compared to the control group $(p<0,05)$ (Fig. 2, 3). The SF-36, BBS and FRT results didn't significantly change in both groups.

Conclusions: The chair-based exercises in frail elderly are effective tool for upper and lower extremities muscles strength, but two months activity are not enough for balance training and life quality changes.

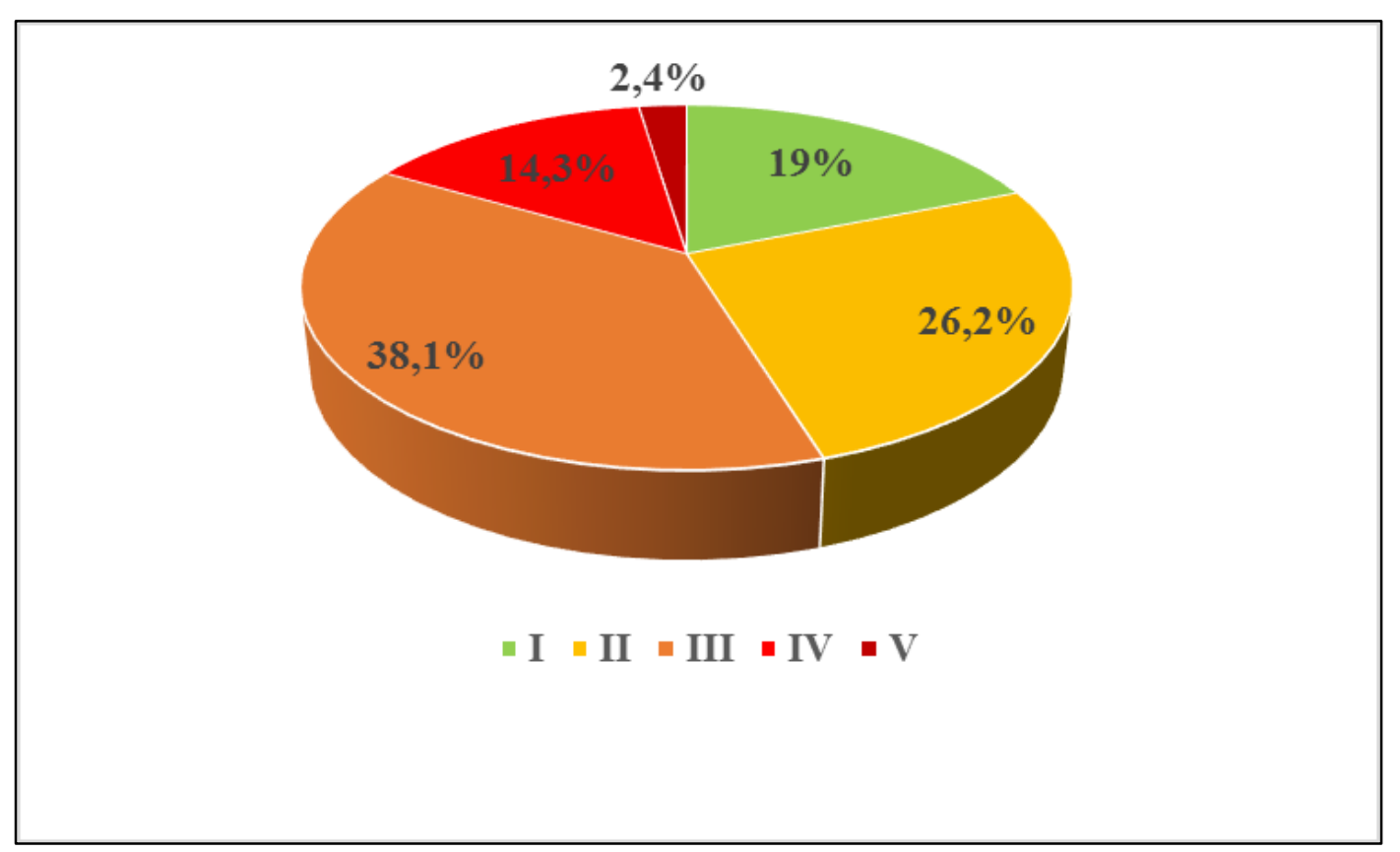

Figure 1. Distribution of participants in accordance with Fried frailty criteria amount, \%

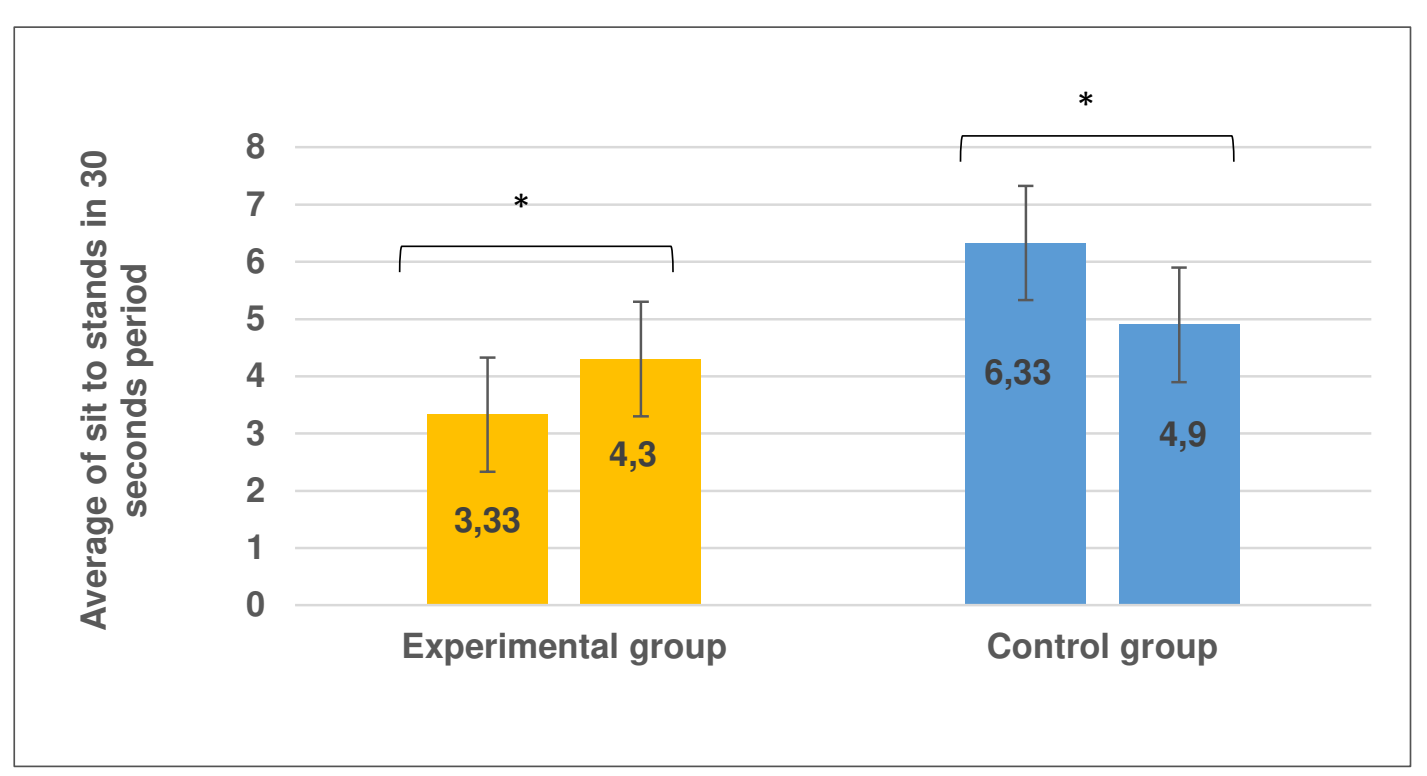

Figure 2. Averages of sit to stands in 30 seconds period in both groups after both evaluations

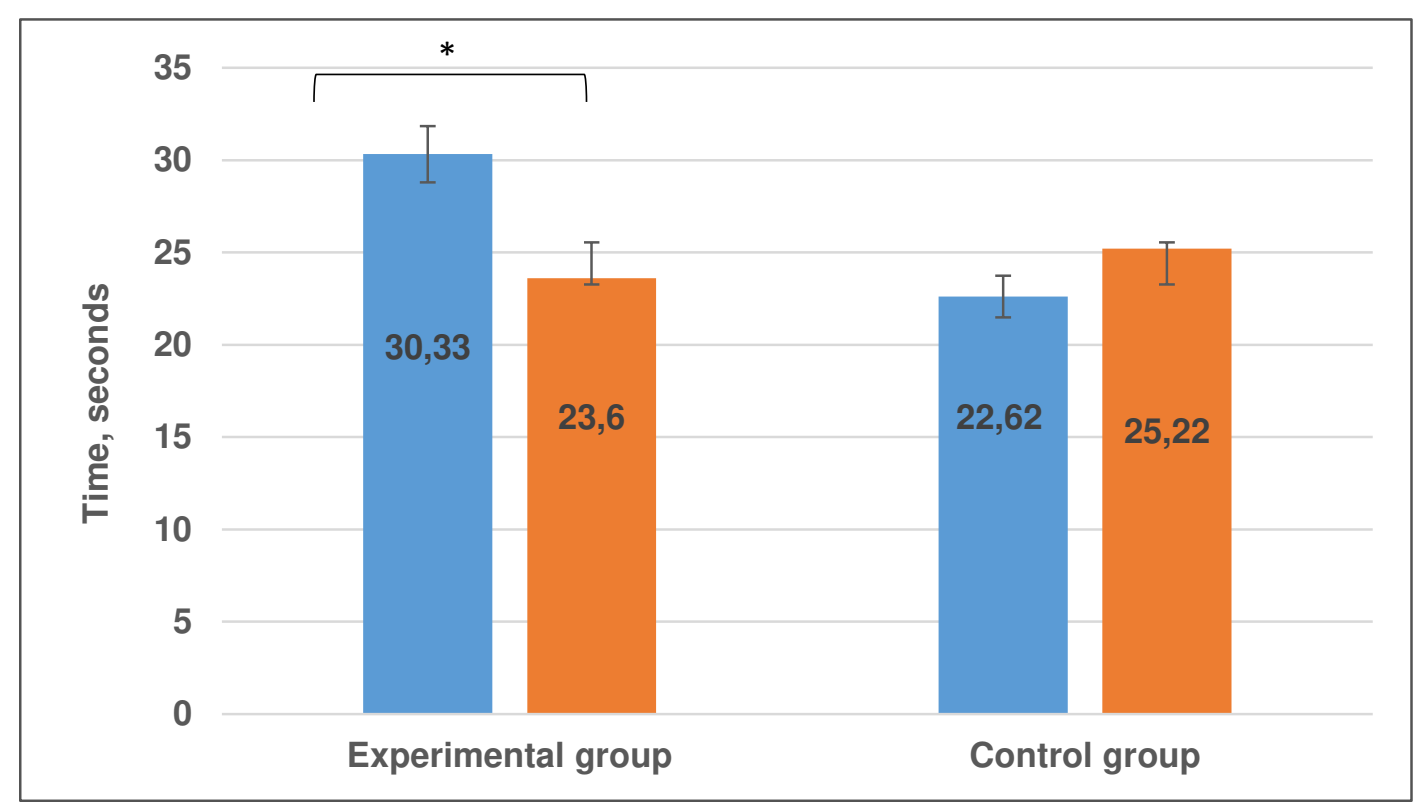

Figure 3. Averages of Timed up and Go test of both groups after two evaluations

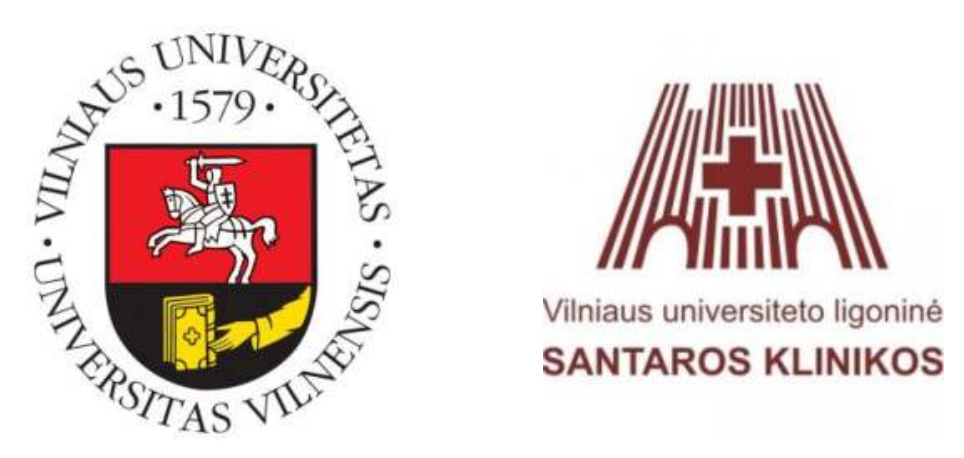

